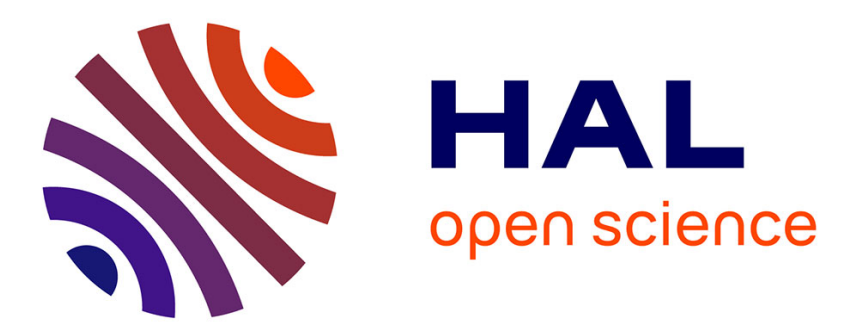

\title{
Currentvoltage characteristics of dendrimer light-emitting diodes
}

S G Stevenson, I D W Samuel, S V Staton, K A Knights, P L Burn, J H T Williams, Alison B Walker

\section{- To cite this version:}

S G Stevenson, I D W Samuel, S V Staton, K A Knights, P L Burn, et al.. Currentvoltage characteristics of dendrimer light-emitting diodes. Journal of Physics D: Applied Physics, 2010, 43 (38), pp.385106. 10.1088/0022-3727/43/38/385106 . hal-00569710

\section{HAL Id: hal-00569710 https://hal.science/hal-00569710}

Submitted on 25 Feb 2011

HAL is a multi-disciplinary open access archive for the deposit and dissemination of scientific research documents, whether they are published or not. The documents may come from teaching and research institutions in France or abroad, or from public or private research centers.
L'archive ouverte pluridisciplinaire HAL, est destinée au dépôt et à la diffusion de documents scientifiques de niveau recherche, publiés ou non, émanant des établissements d'enseignement et de recherche français ou étrangers, des laboratoires publics ou privés. 


\title{
Current-voltage characteristics of dendrimer light-emitting diodes
}

\author{
S.G. Stevenson ${ }^{1}$, I.D.W. Samuel ${ }^{1}$, S.V. Staton ${ }^{2}$, K. A. \\ Knights $^{2}$, P.L. Burn ${ }^{2,3}$,J.H.T. Williams ${ }^{4}$, Alison B. Walker ${ }^{4}$ \\ ${ }^{1}$ Organic Semiconductor Centre, SUPA, School of Physics and Astronomy, \\ University of St Andrews, North Haugh, St Andrews, Fife, KY16 9SS, United \\ Kingdom \\ ${ }^{2}$ Department of Chemistry, Chemistry Research Laboratory, 12 Mansfield \\ Road, Oxford, OX1 3TA, United Kingdom \\ ${ }^{3}$ Centre for Organic Photonics and Electronics, The University of Queensland, \\ Chemistry Building, School of Chemistry \& Molecular Biosciences, Queensland, \\ 4072, Australia \\ ${ }^{4}$ Department of Physics, University of Bath, Bath, BA2 7AY, United Kingdom \\ E-mail: a.b.walker@bath.ac.uk
}

\begin{abstract}
We have investigated current-voltage (I-V) characteristics of unipolar and bipolar organic diodes that use phosphorescent dendrimers as the emissive organic layer. Through simulation of the measured I-V characteristics we were able to determine the device parameters for each device structure studied, leading to a better understanding of injection and transport behaviour in these devices. It was found that the common practice of assuming injection barriers are equal to the difference between bare electrode work functions and molecular orbital levels is unsuitable for the devices considered here, particularly for gold contacts. The studies confirm that different aromatic units in the dendrons can give significant differences in the charge transporting properties of the dendrimers.
\end{abstract}




\section{Introduction}

Organic light-emitting diodes (OLEDs) have attracted much interest due to their potential use in low-cost display technologies [1]. Current display research is focussed on three classes of organic materials: small molecules, polymers and dendrimers. Light-emitting dendrimers consist of a luminescent core, dendrons (branched moieties) and surface groups. The core controls the emission colour, the dendrons control the transport of charge and the surface groups govern the processing properties, thus these properties can be manipulated independently of each other and it is possible to develop a single material in which all three properties are simultaneously optimised. Dendrimers can exploit the possibility of triplet harvesting that occurs in electrophosphorescent devices [2]. By an appropriate choice of surface groups, dendrimers can be synthesised that are readily soluble in many organic solvents and so can be easily solution-processed for device fabrication [3].

The most efficient dendrimers developed so far are those containing a factris (2-phenylpyridyl)iridium(III) [(Irppy $\left.)_{3}\right]$ core, covalently attached phenylene-based dendrons and 2-ethylhexyloxy surface groups. The dendrons are attached to the ligand phenyl ring that in turn is attached to the pyridine ring. An example is the first generation dendrimer, dendrimer $\mathbf{1}$, whose chemical structure is shown in the top panel of Figure 1. This material has been shown to be capable of producing highly efficient OLEDs when blended with suitable hosts $[4,5,6,8]$, and given the correct processing conditions as neat films [3]. However, it has a zero-field charge mobility of only $9.3 \times 10^{-7} \mathrm{~cm}^{2} /(\mathrm{Vs})[4]$.

The present paper has two main aims. One is to develop a model for the currentvoltage characteristics of dendrimer LEDs, and the other is to explore the effect of the choice of contacts and dendrons on charge injection and transport. The latter aspect relates to the fact that LEDs in which the light-emitting dendrimer is blended with carbazole containing hosts are more efficient than those made from a neat dendrimer film [6]. Accordingly we have developed dendrimers which incorporate the host by having carbazole-containing dendrons [9] and an example is dendrimer 2 (Figure 1). The carbazole dendrons lead to much higher hole mobility than biphenyl dendrons for the dendrimers and molecular orbital calculations suggest that this is due to the HOMO being located not only on the core but also on the dendrons allowing holes to hop between dendrons, whereas in the biphenyl case, hole transport is between the cores of the dendrimers [9]. Here, we compare characteristics of single layer devices made from dendrimers $\mathbf{1}$ and 2. For ease of comparison we have selected first generation materials of each type, that is, dendrimers with one level of branching in the dendrons.

Charge carrier injection is critical to the OLED applications mentioned above as it ultimately determines key measures of device operation such as driving voltage and balance of electron and hole injection in OLEDs. To develop reliable device processing conditions, a deep understanding of the mechanisms that determine the electronic properties at the organic-electrode interfaces, i.e. the relative position of molecular levels and charge carrier transport states, is required since contact performance cannot be dissociated from bulk transport properties[10]. In the Schottky-Mott picture, the energy barrier would simply be the difference between the work function of the contacts and the relevant energy level of the organic semiconductor. However, the present work is motivated by photoemission measurements of charge injection barriers in devices made of small organic molecules [11] that have shown that a contact made 


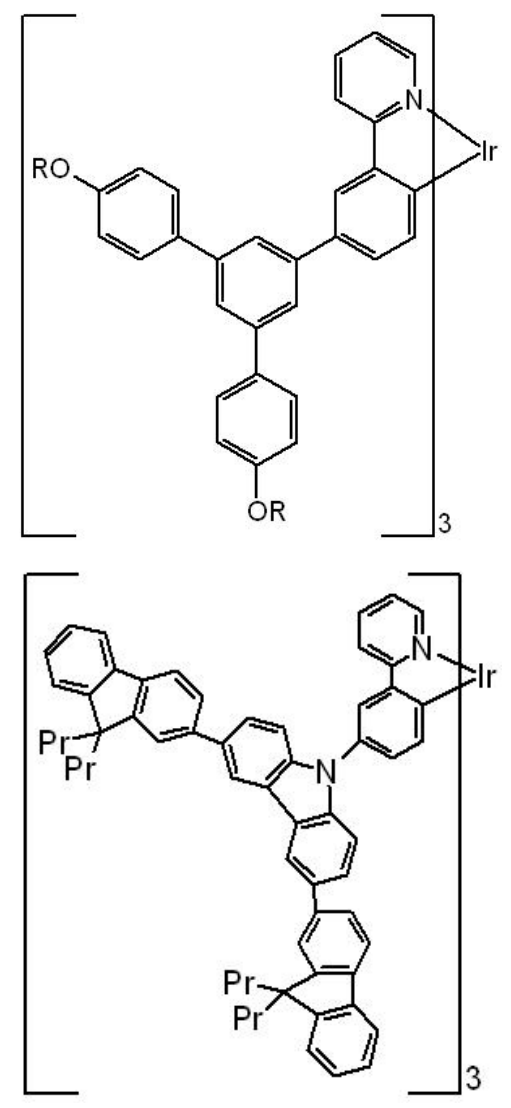

Figure 1. Structure of dendrimer 1 (top) where $\mathrm{R}=2$-ethylhexyl, dendrimer $\mathbf{2}$ where $\operatorname{Pr}=$ n-propyl (bottom)

under commonly used conditions for organic devices is not 'ultraclean' but contains a layer of adsorbed molecules. This layer reduces the surface dipole at the contact by an amount large enough that the charge injection barrier is insensitive to the underlying metal [12]. We therefore explore the effect of different electrode materials on the apparent barrier to charge injection. Additionally, we investigate differences in electrical behavior between organic-on-metal and metal-on-organic contacts as such differences have been explained from seeing how the interface is created [10].

We have developed a drift-diffusion model that can describe charge injection and transport behaviour including space charge effects within the device [13]. This approach is a considerable advance on earlier models that neglect injection [14]. Due to the importance of charge injection, we have included a recent formulation of this process specifically adapted to organic materials [15]. We find that the fitted barrier heights differ noticeably from their predicted values based on the measured dendrimer HOMO and LUMO levels and metal work functions. We have focussed mainly on hole-only devices to reduce the number of fitting parameters required for our model. Bipolar devices for the two dendrimers were also made using a calcium top contact. The disperse nature of electron transport in these systems means that electrons do not have a well defined mobility and hence the use of a bipolar drift diffusion model is 
suspect. Nevertheless, we were able to obtain an electron barrier height by fitting to an expression for the tunneling current at high bias and to obtain the I-V characteristics for the bipolar device structure ITO/2/Ca-Al.

The layout of this paper is as follows: we describe the experiments and model used, then present and discuss our results and finally summarise our findings.

\section{Experiment}

Dendrimers $\mathbf{1}$ and $\mathbf{2}$ are readily soluble in polar aprotic organic solvents and so could be easily solution-processed for device fabrication $[5,6,7]$. We spin-coated a number of films of each dendrimer from chloroform solutions at concentrations of $20 \mathrm{mg} / \mathrm{ml}$ yielding films approximately $100 \mathrm{~nm}$ thick onto the desired substrate. In all cases prior to film deposition the substrates underwent a routine cleaning procedure of washing in ultrasonic baths of acetone, chloroform and 2-propanol followed by oxygen plasma ashing at $100 \mathrm{~W}$ for five minutes. The cleaned substrates were then used to construct devices of various geometries. In each case a basic sandwich structure of a bottom contact, spin-coated dendrimer layer, and a top metal contact was used.

A range of contact materials were used to allow the effect of the work function of the contacts to be explored. Transparent indium tin oxide (ITO), gold (Au) or platinum $(\mathrm{Pt})$ were the bottom contact materials. Top contacts were made from aluminium (Al), Au or Pt. To investigate the effect of enhanced electron injection into the organic layer, devices with a calcium $(\mathrm{Ca})$ top contact capped with $\mathrm{Al}$ to avoid oxidation were also made. All electrode metals were deposited at a rate in the range $0.1-0.3 \mathrm{~nm} /$ second depending on the metal used at pressures typically in the region of $3 \times 10^{-6}$ mbar. Prior to spin-coating of the dendrimer, the gold bottom contact was cleaned by the method of Scott et al. [16], viz by washing in the spinning solution or by a short oxygen plasma ash, but we did not observe any difference in the device characteristics between these methods. After spin-coating the devices were transferred back to the evaporator where a metal top contact was deposited to define a device area of $\sim 10^{-2} \mathrm{~cm}^{2}$. On removal from the evaporator the devices were immediately characterised using a Keithley 2400 source measure unit in the dark and in air.

\section{Model}

In earlier modelling studies on devices containing dendrimer 1 [4], the model of Davids et al. [17] was used to describe device behavior. Here we employ a model that, as described in [13], self-consistently solves the coupled time-independent electron and hole continuity equations, and Poisson's equation, thus making full allowance for space charge effects.

The current continuity equations for the free electron and hole densities $n$ and $p$ respectively, noting that $n$ and $p$ are dominated by injected carriers since there are few intrinsic carriers, are

$$
\frac{\mathrm{d} J_{n}}{\mathrm{~d} x}=-q(G-R), \quad \frac{\mathrm{d} J_{p}}{\mathrm{~d} x}=q(G-R) .
$$

Here $J_{n}$ and $J_{p}$ are the electron and hole current densities respectively, $G$ is the electron-hole pair generation rate (taken to be zero), and $q$ is the magnitude of the electric charge. $R$ is the net recombination rate and has contributions from 
both trap-related and exciton formation processes. The exciton formation rate (here called the optical recombination rate, $R_{\text {opt }}$, has a bimolecular form with a Langevin recombination coefficient, $\gamma_{L}$ :

$$
R_{o p t}=\gamma_{L}\left(n p-n_{i n t}^{2}\right), \gamma_{L}=\frac{4 \pi q \mu_{R}}{\epsilon}
$$

where $n_{\text {int }}$ is the intrinsic carrier density, $\mu_{R}$ is an effective recombination mobility, taken to be the larger of the electron and hole mobilities in the material, and $\epsilon$ is the dielectric constant of the material.

Poisson's equation relates the electrostatic potential, $\psi$, with the charge in the device,

$$
\frac{\mathrm{d}^{2} \psi}{\mathrm{d} x^{2}}=-\frac{q}{\epsilon}(p-n)
$$

The equations relating $n$ and $p$ to the electrostatic potential are derived from the Maxwell-Boltzmann distribution;

$$
\begin{aligned}
& n=N_{C} \exp \left[\frac{q}{k_{B} T}\left(\psi-\phi_{n}+\frac{\chi_{c}}{q}\right)\right] \\
& p=N_{V} \exp \left[-\frac{q}{k_{B} T}\left(\psi-\phi_{p}+\frac{E_{g}}{q}+\frac{\chi_{c}}{q}\right)\right]
\end{aligned}
$$

where $\phi_{n}$ and $\phi_{p}$ are the electron and hole quasi-Fermi levels respectively, and $\chi_{c}$ is the electron affinity of the material.

A sophisticated treatment of the current at the contacts [15] is adopted. This treatment is a development of the well-known work by Scott and Malliaras [18]. There are two contributions to the current across the interface: injection of electrons from the cathode to the acceptor and vice versa. This approach is based on the assumption that the surface recombination of charge carriers with their image charges is analogous to the Langevin bimolecular recombination in amorphous semiconductors. In the Langevin theory, the average electron/hole pair recombines if their Coulombic binding energy exceeds $k_{B} T$ where $k_{B}$ is the Boltzmann constant and $T$ the absolute temperature. That happens when the distance between them is the so-called Coulomb radius $r_{c}=q^{2} /\left(4 \pi \epsilon k_{B} T\right)$. Injection takes place through thermionic emission over a Schottky barrier $\phi_{B p}$ for hole injection and $\phi_{B n}$ for electron injection. The barrier reduction at an organic metal interface due to the image charge potential is accounted for.

For charge transport, the widely used Poole-Frenkel form for the field-dependent carrier mobility in the presence of shallow traps,

$$
\mu=\mu_{0} \exp (\gamma \sqrt{E}),
$$

was adopted, where $\mu_{0}$ is the zero-field mobility, $E$ is the electric field strength and $\gamma$ is a constant determining the field dependence of the mobility. Deep traps have not been considered as we had no clear evidence for them so had no information about their concentration and depth and needed to minimise the number of adjustable parameters.

We introduced a series resistance, $R_{I}$, as a fitting parameter as was done in [19] for reasons discussed in the following section. To determine $R_{I}$, a current-voltage characteristic in which $\phi_{B p}$ had been varied to obtain the best fit was generated with $R_{I}=0$. This characteristic showed how the current $I$ varies with the voltage across the dendrimer layer, $V_{\text {dend }}$. The applied bias $V_{a}$ was modified to allow for an Ohms 
law drop across $R_{I}$ through the expression $V_{a}=V_{\text {dend }}+I R_{I}$ and the characteristic $I$ vs $V_{a}$ obtained for trial values of $R_{I}$.

The thickness of the dendrimer layer was measured as $100 \mathrm{~nm}$, and we assume an ambient temperature of $300 \mathrm{~K}$. In the absence of measurements of the dielectric permittivity, a value of 3.0 typical for organic semiconductors was assumed. As is standard in drift diffusion models of organic semiconductors, we equate the LUMO level to the conduction band minimum and the HOMO level to the valence band maximum. Here, we took the conduction and valence band densities of states each to be $10^{27} \mathrm{~m}^{-3}$. As pointed out by [20], the results from this model are insensitive to this value.

We fit our predicted characteristics to experimental data by varying $\phi_{B p}$ and fixing $\mu_{0}$ and $\gamma$ to experimental values to reduce the number of fitting parameters. The fitting procedure was to compare the data and the fit by eye. For dendrimer $1 \mu_{0}$ and $\gamma$ were fixed respectively to $9.3 \times 10^{-7} \mathrm{~cm}^{2} /(V s)$ and $3.4 \times 10^{-3} \mathrm{~cm}^{1 / 2} / \mathrm{V}^{1 / 2}$ [4]. These values for $\mu_{0}$ and $\gamma$ were obtained by modelling using the model of Davids et al.[17] and as discussed in [4] agree well with experimental time of flight measurements. For dendrimer 2, time of flight measurements gave $1.2 \times 10^{-5} \mathrm{~cm}^{2} /(\mathrm{Vs})$ and $3.0 \times$ $10^{-3} \mathrm{~cm}^{1 / 2} / \mathrm{V}^{1 / 2}$ respectively [21]. Dendrimers can be synthesized such that they are monodisperse so that there is strong reproducibility for different batches. Measurements using the methodology described in [4] are on devices whose thickness is similar to the devices studied in this paper rather than the micron thicknesses usually required for time of flight measurements. Transport is only weakly dispersive at room temperature so that mobilities are not sensitive to film thickness and it is carefully argued in [4] and [21] that their values of $\mu_{0}$ and $\gamma$ should be applicable to devices made from dendrimer 1. Furthermore, references [4] and [21] are the only reports of mobility measurements made for these materials to the authors' knowledge, the measurements are made in the direction perpendicular to the plane of the film (in contrast to FET based measurements), which is the direction relevant to OLED operation and the measurements were made in the same experimental group as for the submitted paper, ensuring consistency of film preparation as far as possible.

\section{Results and Discussion}

We will begin by considering hole-only devices of dendrimer $\mathbf{1}$. Table 1 summarises the device structures studied, and representative fits to the experimental current-voltage characteristics are shown in Figure 2. Our fits are not good at low bias since in an effort to establish the main physics determining carrier transport and to avoid the charge of 'fitting an elephant' we have only a few parameters.

We first consider devices with ITO as the injecting contact whose characteristics are shown in the top panel of Figure 2. For the device ITO/1/Al we fit a barrier to charge injection of $0.4 \mathrm{eV}$, and find a series resistance of $1200 \Omega$. This barrier is compatible with that expected from Schottky-Mott theory, after taking account of the errors in estimates of the energy of the dendrimer HOMO, and the ITO workfunction, which depends on the details of its preparation [22]. No electron injection from the $\mathrm{Al}$ contact is expected in this device because of the large difference between the dendrimer LUMO at $2.5 \mathrm{eV}$ and the $\mathrm{Al}$ workfunction of $4.3 \mathrm{eV}$. The absence of electron injection was confirmed by the fact that no light emission was detected from the device. When $\mathrm{Au}$ or Pt were used as the top contact, the current through the device was smaller, possibly by creating a barrier to hole extraction in the devices or because they damage 

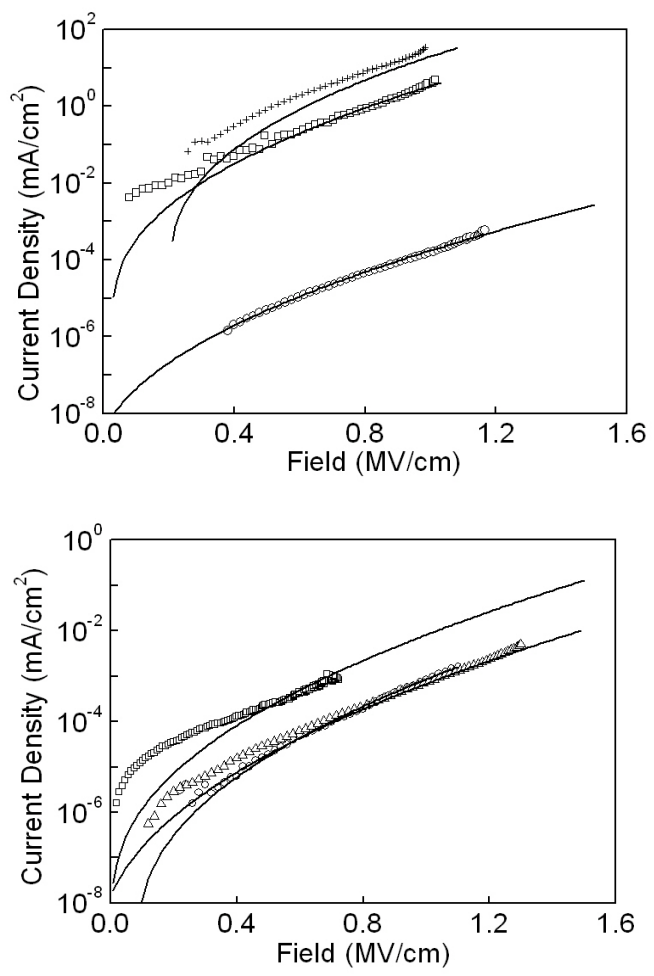

Figure 2. Current density vs field characteristics for dendrimer $\mathbf{1}$ with an injecting contact of ITO (top panel), Au (bottom panel). Top panel: $\underline{\mathrm{ITO}} / \mathbf{1} / \mathrm{Al}$ $(+), \underline{\mathrm{ITO}} / \mathbf{1} / \mathrm{Au}(\square), \underline{\mathrm{ITO}} / \mathbf{1} / \mathrm{Pt}(\mathrm{O})$. Bottom panel: $\underline{\mathrm{Au}} / \mathbf{1} / \mathrm{Al}(\mathrm{O}), \underline{\mathrm{Au}} / \mathbf{1} / \mathrm{Au}$ $(\square), \underline{\mathrm{Au}} / \mathbf{1} / \mathrm{Pt}(\triangle)$. Symbols, experimental data, solid lines, modelling data. The injecting contact material is underlined.

\begin{tabular}{cccc}
\hline Structure & $\phi_{B p}(\mathrm{eV})$ & $\phi_{B p}^{(S M)}(\mathrm{eV})$ & $R_{I}(\Omega)$ \\
\hline$\underline{\mathrm{ITO}} / \mathbf{1} / \mathrm{Al}$ & 0.4 & 0.5 & $1.2 \times 10^{3}$ \\
$\underline{\mathrm{ITO}} / \mathbf{1} / \mathrm{Au}$ & 0.48 & 0.5 & $7 \times 10^{3}$ \\
$\underline{\mathrm{ITO}} / \mathbf{1} / \mathrm{Pt}$ & 0.74 & 0.5 & $7 \times 10^{7}$ \\
$\underline{\mathrm{Au}} \mathbf{1} / \mathrm{Al}$ & 0.69 & 0.4 & 0 \\
$\underline{\mathrm{Au}} / \mathbf{1} / \mathrm{Au}$ & 0.64 & 0.4 & $1.1 \times 10^{8}$ \\
$\underline{\mathrm{Au}} / \mathbf{1} / \mathrm{Pt}$ & 0.7 & 0.4 & $5 \times 10^{6}$ \\
$\mathrm{Au} / \mathbf{1} / \underline{\mathrm{Pt}}$ & 0.67 & 0.1 & $7 \times 10^{6}$ \\
\hline
\end{tabular}

Table 1. For the measured device structures shown, the hole injection barrier heights obtained from simulation $\phi_{B p}$ are compared with values deduced from the Schottky-Mott model, $\phi_{B p}^{(S M)}=E_{H O M O}-\phi_{m}$ where $\phi_{m}$ is the metal workfunction and $E_{H O M O}$ the HOMO level. To find $\phi_{B p}^{(S M)}, E_{H O M O}$ was assumed to be $5.6 \mathrm{eV}$ below the vacuum, the workfunctions of treated ITO, clean $\mathrm{Au}$, clean Pt, Al to be respectively $5.1 \mathrm{eV}$ [23], $5.2 \mathrm{eV}$ [12], $5.5 \mathrm{eV}$ [12], $4.3 \mathrm{eV}$ [24]. The fitted value of the series resistance $R_{I}$ is also shown. The injecting contact is underlined. 
the surface and near surface region of the dendrimer film due to their high kinetic energy during thermal deposition. This reduction in current does not arise from space charge effects since as noted above, these effects are included in our model. The only way that the reduced current could be modelled was by increasing the fitted barrier height for the bottom (hole injecting) contact and the series resistance.

As there is a significant barrier to hole injection from ITO we next explored the effect of changing the bottom contact. The Schottky-Mott model would suggest that replacing ITO by $\mathrm{Au}$ would reduce the barrier to charge injection, and so increase the current through the LEDs. The results of this change are shown in Figure 2(bottom). The gold bottom contact was investigated in devices with $\mathrm{Al}, \mathrm{Au}$ and Pt top contacts. Comparing the characteristics of these devices shown in the bottom panel of Figure 2 with the results for the device of structure ITO/1/Al in the top panel of Figure 2 shows that the currents are approximately four orders of magnitude smaller. Furthermore, the fitted barrier heights are substantially higher than for the ITO/1/Al device. Together these results suggest that gold does not give the expected reduction of injection barrier. This reduction in current shows that the interface between the organic and the gold layer is less effective at charge injection than an oxygen plasma ashed ITO-dendrimer interface. In previous reports $[11,25,26]$, the anomalous behaviour of polymer-gold interfaces has been explained by the presence of interface dipoles caused by the repulsion between the electrons of the organic and the metal layer. This leads to a suppression of the metal electron density tail, which acts to lower the metal work function and hence increase $\phi_{B p}$ as we have found here.

We found that varying $\phi_{B p}$ and $\mu_{0}$, to a very good approximation, only affects the magnitude of the current, not the gradient [20, 27]. As a general rule, it has been found that an increase in the injection barrier height by $0.1 \mathrm{eV}$ has a similar effect on the current magnitude as decreasing the hole mobility, namely changing the current magnitude by a factor of 10 at all biases. To vary the gradient of the current with respect to the voltage, $\gamma$ could be varied as was done in [20]. However, as this parameter is known for both materials under consideration, $\gamma$ is kept constant throughout and an equivalent circuit series resistance, $R_{I}$, is introduced [19] which was found once the best value of $\phi_{B p}$ had been determined. Thus the correlation between $\phi_{B p}$ and $R_{I}$ is not close. If it were close, it would not be necessary to introduce $R_{I}$.

In [19] we showed that in such a case, experimental data could be understood with our model if a resistive interfacial region or resistive contact were inserted in series with the device. Since the device areas are all $\sim 10^{-2} \mathrm{~cm}^{2}$, the values found for $R_{I}$ per unit area for the ITO bottom contacts are comparable to our previous results for conjugated polymers [19] $\left(\sim 10^{4} \Omega \mathrm{cm}^{-2}\right)$. As the barrier heights for hole injection are in most cases considerably higher for the Au bottom contacts, the current in the devices is lower and there is therefore a smaller voltage dropped across $R_{I}$.

Our fitted $R_{I}$ values shown in Table 1 varied from zero for the $\underline{A u} / \mathbf{1} /$ Al device to $1.1 \times 10^{8}$ for the $\underline{A u} / \mathbf{1} / A u$ device. The only trend that can be deduced from this data is that the Al top contacts show much lower values of $R_{I}$ than Au or Pt top contacts. This observation suggests that there may be contaminants in or on the Au or Pt top contacts that are blocking the extraction of holes from the device.

We have also studied a device with a Pt injecting electrode. We studied two such structures: $\underline{\mathrm{Au}} / \mathbf{1} / \mathrm{Pt}$ in forward bias and $\mathrm{Au} / \mathbf{1} / \underline{\mathrm{Pt}}$ in reverse bias. Schottky-Mott theory predicts that these devices should have ohmic hole injection, and identical behaviour. The similarity in values of the injection barrier heights for the forward and reverse biases is encouraging. However, the modelled current for devices with 


\begin{tabular}{cccc}
\hline Structure & $\phi_{B p}(\mathrm{eV})$ & $\phi_{B p}^{(S M)}(\mathrm{eV})$ & $R_{I}(\Omega)$ \\
\hline$\underline{\mathrm{ITO}} / \mathbf{2} / \mathrm{Al}$ & 0.68 & 1.0 & $1 \times 10^{5}$ \\
$\underline{\mathrm{ITO}} / \mathbf{2} / \mathrm{Au}$ & 0.54 & 1.0 & $7 \times 10^{5}$ \\
$\underline{\mathrm{Au}} / \mathbf{2} / \mathrm{Au}$ & 0.80 & 0.5 & 0 \\
$\underline{\mathrm{Au}} / \mathbf{2} / \mathrm{Al}$ & 0.74 & 0.5 & 0 \\
\hline
\end{tabular}

Table 2. Parameter values for dendrimer $\mathbf{2}$ devices. The HOMO level in dendrimer 2 was set at $5.7 \mathrm{eV}$ below the vacuum [9].

ohmic contacts gave currents which were significantly too high, which could not be fitted satisfactorily, even with a large $R_{I}$. The reason for the poor device performance associated with a Pt electrode is unclear. The addition of a series resistance into the circuit only improves the fit at high values of the applied bias where the current is highest, and therefore a larger fraction of the applied potential will be dropped across the resistive layer.

The fitted device parameters for dendrimer $\mathbf{2}$ are listed in Table 2 and representative current-voltage characteristics, together with their fits are shown in Figure 3. We first consider devices with ITO injecting contacts. Figure 3 shows that for these devices with either an $\mathrm{Al}$ or $\mathrm{Au}$ top contact, the current density is smaller than the corresponding devices made from dendrimer 1. The fits to the data suggest that the lower current is due to a higher barrier to charge injection and (in the case of the gold bottom contact) a higher series resistance. Figure 3 also shows the results for devices of dendrimer $\mathbf{2}$ with an Au bottom contact. The current is lower than for an ITO bottom contact, and lowest when the top contact is also Au. As is the case for dendrimer $\mathbf{1}$, the current is smaller for an Au bottom contact than an Al bottom contact. The fits show that the lower current associated with Au bottom contacts is due to a higher barrier height, even though the Schottky-Mott picture would suggest the barrier to hole injection from gold should be lower.

In addition to the hole-only devices of dendrimers $\mathbf{1}$ and $\mathbf{2}$ described above, we have also studied electron transport in order to develop a bipolar device model. Electron only devices consisting of a layer of a Ca bottom contact and top contact with a layer of dendrimer $\mathbf{1}$ or dendrimer $\mathbf{2}$ in between were made. The top contact was also coated with a layer of $\mathrm{Al}$ to slow oxidation. The current-voltage characteristics were measured. Ca has a vacuum work function of $2.9 \mathrm{eV}$ [28], and the dendrimer LUMOs are $2.5 \mathrm{eV}$, so according to the Schottky-Mott picture, the barrier to charge injection would be in the region of $0.4 \mathrm{eV}$. The applied biases used in these devices were run up to $50 \mathrm{~V}$ compared with $15 \mathrm{~V}$ for the hole-only devices discussed so far.

With the large values of the applied bias in these electron-only cases, it is likely that tunneling is the dominant component of the current. The change in the gradient of the current-voltage characteristic at high bias denotes the onset of tunneling. The height of the injection barrier can be obtained using a Fowler-Nordheim graph [29], the gradient of which, $\kappa$, gives the injection barrier height from

$$
\kappa=\frac{8 \pi\left(2 m^{*}\right)^{1 / 2} \phi_{B p}^{3 / 2}}{3 h e},
$$

where $m^{*}$ is the electron effective mass (the free electron mass was used here), $\phi_{B p}$ is the height of the barrier and $h$ is Planck's constant. The Fowler-Nordheim plots 


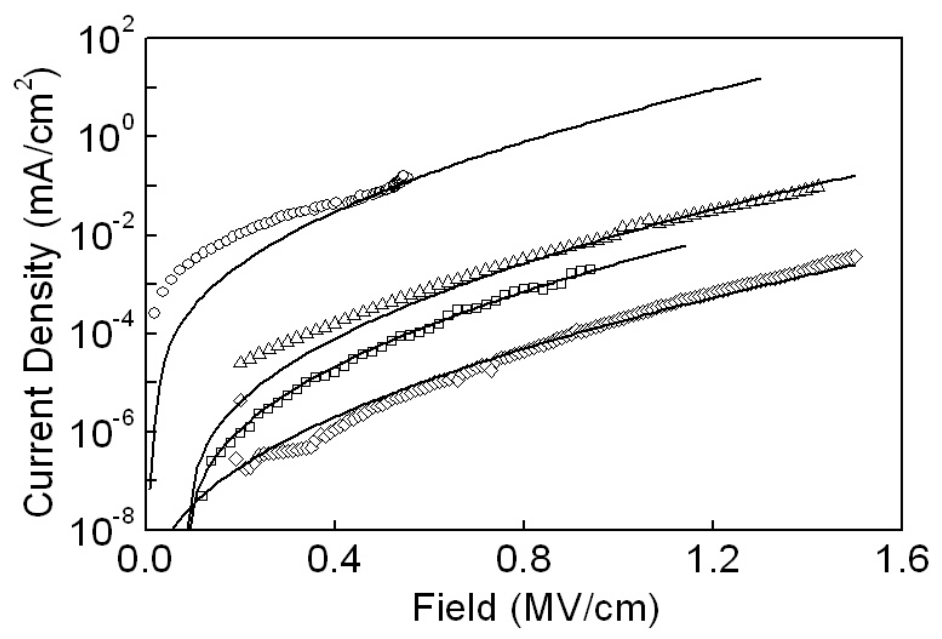

Figure 3. Current density $v s$ field for devices made from dendrimer $\mathbf{2}$ with different electrode materials: $\underline{\mathrm{ITO}} / \mathbf{2} / \mathrm{Al}(\triangle), \underline{\mathrm{Au}} / \mathbf{2} / \mathrm{Al}(\square), \underline{\mathrm{Au}} / \mathbf{2} / \mathrm{Au}(\diamond)$, ITO/2/Au $(\bigcirc)$. Symbols, experimental data, solid lines, modelling data. The injecting contact material is underlined.

for the devices considered here are shown in Figure 4 and give $0.6 \mathrm{eV}$ and $0.3 \mathrm{eV}$ for the barrier height for electron injection into dendrimers $\mathbf{1}$ and $\mathbf{2}$ respectively. This difference cannot be explained by the Schottky-Mott theory since the two materials should have a similar LUMO energy level as the LUMO molecular orbital distribution is the same [9] hence a similar electron injection barrier height. Tunneling injection may not be the only mechanism as the Fowler-Nordheim plot for the dendrimer $\mathbf{1}$ device is less linear than for the dendrimer $\mathbf{2}$ device. However, the reason for using this procedure to determine the barrier height is to avoid introducing additional fitting parameters into the model.

The barrier heights obtained were used in a bipolar model. In the absence of more information, e.g. temperature dependent characteristics, the same value of $\gamma$ was used for electrons and holes and the zero-field electron mobility $\mu_{n 0}=10^{-2} \mu_{p 0}$. Measured and fitted dendrimer 2 bipolar I-V characteristics are compared in Figure 5. Our fitted current has the correct order of magnitude for bias voltages between 6 and $8 \mathrm{~V}$ although the gradient of the curve is too low for higher biases, suggesting that the mobility field dependence parameter, $\gamma_{n}$, is greater than the value we adopted, namely the experimentally determined $\gamma_{p}$ as used in the hole-only device simulations. We could not fit the data for the dendrimer $\mathbf{1}$ device with our model, the current being roughly a factor of 100 too low, thus the electron injection barrier height obtained from the Fowler-Nordheim plots is too large.

The experimental current and external quantum efficiency (EQE) for the bipolar device structures are shown in Figure 6. The resulting currents are found to be the greatest measured for any of the devices with either dendrimer, indicating the presence of a significant number of injected electrons. In OLEDs, high efficiency requires balanced injection and transport [30]. We see, for example, at a standard brightness of $100 \mathrm{~cd} / \mathrm{m}^{2}$ dendrimer $\mathbf{1}$ attains an external quantum efficiency of 0.21 


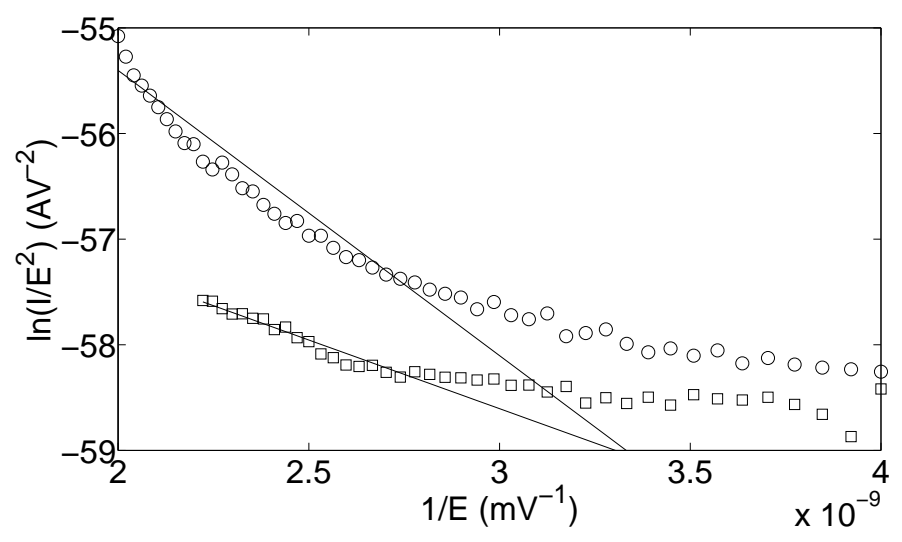

Figure 4. Fowler-Nordheim graphs for electron-only devices with $\mathrm{Ca}-\mathrm{Al}$ injecting contacts for dendrimer $\mathbf{1}(\bigcirc)$ and dendrimer $\mathbf{2}(\square)$. The solid lines indicate the region at high electric field where Equation 7 is assumed to be valid.

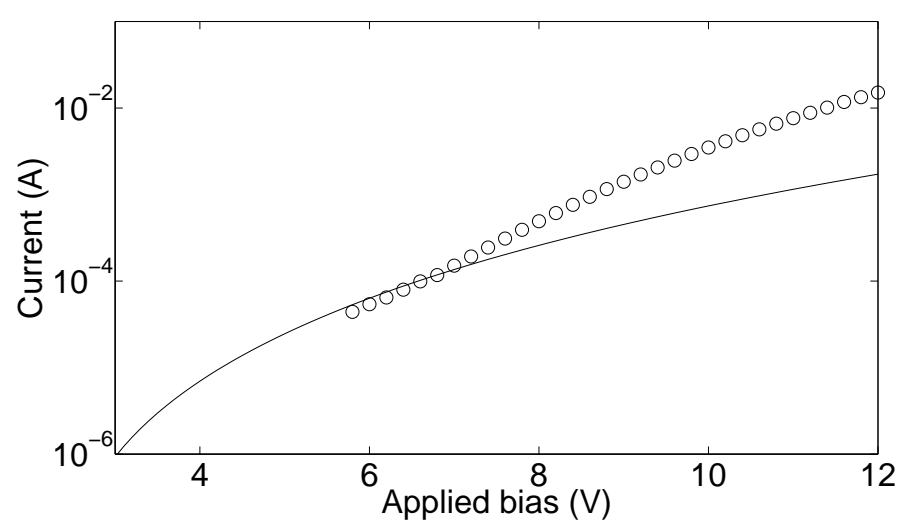

Figure 5. Experimental $(\bigcirc)$ and simulated (solid line) I-V characteristics for the device structure ITO/2/Ca-Al

\% (at $6.0 \mathrm{~V}$ ) whereas at the same brightness, dendrimer 2 is only $0.14 \%$ (at $8.8 \mathrm{~V}$ ) efficient.

Despite the lower mobilities, the total current for the dendrimer $\mathbf{1}$ device is higher than for the dendrimer 2 device. As noted above, a higher barrier height is obtained for electron injection for the dendrimer $\mathbf{1}$ device from the Fowler-Nordheim plot. A possible cause of the higher current for the dendrimer $\mathbf{1}$ device is a reduced hole barrier height at the bottom contact-dendrimer 1 interface. Much higher device efficiencies of $16 \%$ [6] have been achieved with dendrimer 1 in a blend and improving charge balance with the aid of an additional electron transport/hole blocking layer.

Reference [31] showed that in single carrier devices the injection of holes into OLEDs based on F8BT was improved by the addition of an extraction barrier for electrons. This barrier causes the electron density to build up, which increases the electric field at the interface and aids hole injection. Figure 7 shows that the electron density exhibits a peak at the bottom contact (left hand side) even though there is no 

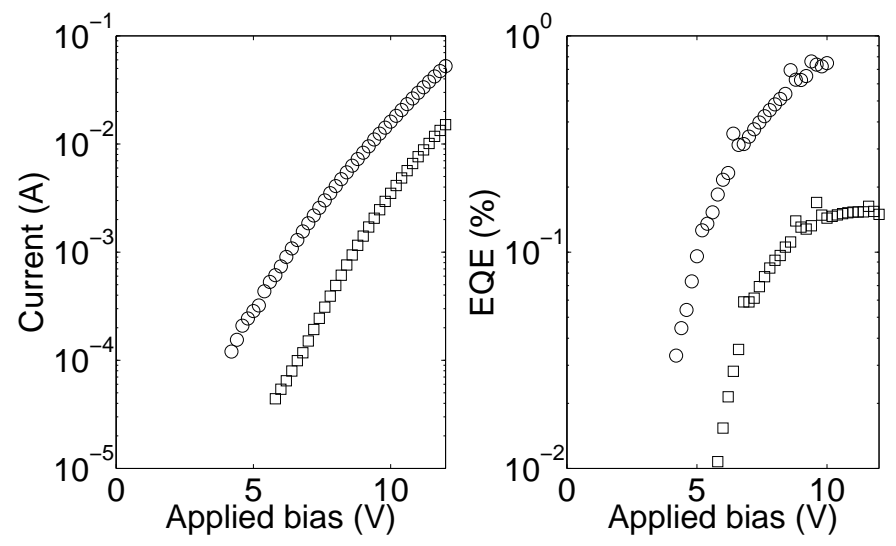

Figure 6. Experimental I-V and EQE characteristics for the devices ITO/dendrimer/Ca-Al, dendrimer $1(\bigcirc)$, dendrimer $2(\square)$. The device area is $0.1 \mathrm{~cm}^{2}$ for both devices.

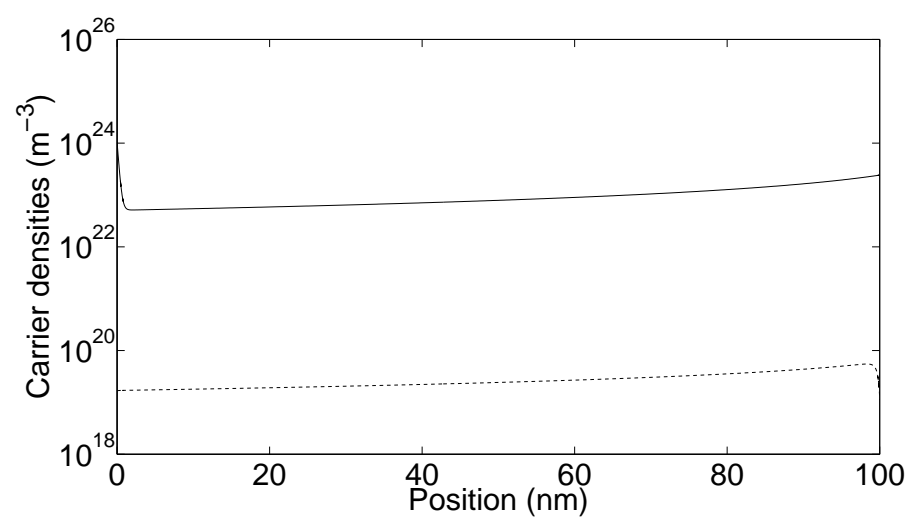

Figure 7. Electron density (solid line), hole density (dashed line) in units of $\mathrm{m}^{-3}$ vs distance from bottom contact in $\mathrm{nm}$ for the structure ITO/dendrimer $2 / \mathrm{Ca}-\mathrm{Al}$ at $12 \mathrm{~V}$ bias. The $x$-axis shows the distance from the bottom contact in $\mathrm{nm}$.

barrier for extraction as considered in [31]. The reason for this is that the extraction rate for charge carriers in the simulation scheme considered here is proportional to the carrier mobility [15]. Since we assumed the electron mobility to be only $1 \%$ of that of the holes, the electrons tend to accumulate at the extracting electrode. The fall in the hole density at the other side of the device in Figure 7 is conversely due to the larger value of the hole mobility. In the absence of space charge effects, the electric field would be equal to $100 \mathrm{MVm}^{-1}$ uniformly across the device. However, as shown in Figure 8, the pile up in electron density at the bottom contact does not greatly increase the field and so is unlikely here to have a large influence on the electron current.

Given the insensitivity of the hole barrier height to the change in the electrode material found above for both dendrimers, the barrier heights for the measured I-V characteristics were recalculated without a series resistance to cut down on the number of adjustable parameters in the model. Although some of the fits became worse, it 


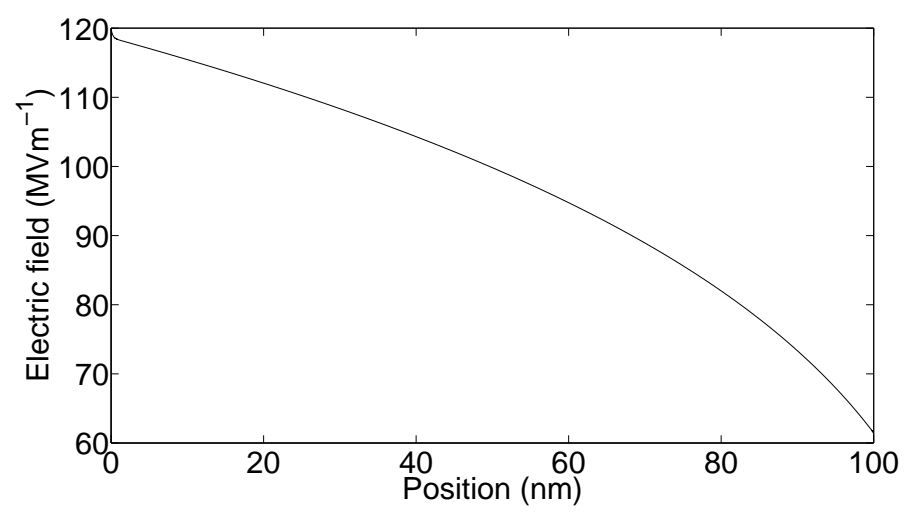

Figure 8. Electric field $\left(\mathrm{MVm}^{-1}\right)$ profile for the structure ITO/dendrimer $\mathbf{2} / \mathrm{Ca}$ $\mathrm{Al}$ at $12 \mathrm{~V}$ bias. The $x$-axis shows the distance from the bottom contact in $\mathrm{nm}$.

enabled us to get a broad overview of the variation with electrode material. The values of injecting electrode work functions were found to lie in the range $5 \pm 0.1 \mathrm{eV}$, with only 3 values, namely $4.8,5.25,5.3 \mathrm{eV}$, outside this range.

Recent work by Grobosch et al. has considered a similar experimental result [12] for the hole injection barrier at the interface between the oligomer $\alpha$-sexithiophene (6T) and the metals $\mathrm{Ag}, \mathrm{Pd}, \mathrm{Au}$ and Pt. The vacuum work functions of these metals vary by more than $1 \mathrm{eV}$ but reference [12] shows that the resultant injection barrier varies by no more than $\sim 0.2 \mathrm{eV}$. Our work shows that this effect may be even more pronounced than in reference [12] since we have shown small variations in the barrier heights for metals whose work functions vary by more than $2 \mathrm{eV}$.

\section{Conclusions}

We have performed a detailed analysis of current-voltage characteristics of single and two carrier devices that use phosphorescent dendrimers as the organic layer. Dendrimer 1 contained phenylene dendrons and dendrimer $\mathbf{2}$ used charge transporting carbazole dendrons. With these two dendrimers devices were fabricated, employing ITO, $\mathrm{Au}$ and $\mathrm{Pt}$ as the bottom contact and $\mathrm{Al}, \mathrm{Au}, \mathrm{Pt}$ and $\mathrm{Ca}-\mathrm{Al}$ top contacts. Results from a full device model were fitted to the current-voltage characteristics to deduce key parameters such as the height of the injection barrier at the injecting contact. Whilst many drift diffusion models of organic devices have been published, it is rare for such models to include space charge effects and a full treatment of charge injection, and thus for comparisons to be possible with a comprehensive set of experimental data that looks at devices made from the same materials with different contacts. We have shown that the fitted barrier heights do not agree with the Mott-Schottky model and are sensitive to the preparation conditions. Our work has confirmed important recent photoemission measurements indicating that the resultant injection barrier varies by no more than $\sim 0.2 \mathrm{eV}[12]$, regardless of the underlying metal. Our overall conclusion is that it is extremely important to model charge injection carefully when deducing device parameters such as charge mobilities from current-voltage characteristics in line with the conclusions of a recent review [10]. 


\section{Acknowledgements}

The authors are grateful to the Engineering and Physical Sciences Research Council,

Scottish Higher Education Funding Council, Cambridge Display Technology and the European Commission STREP project MODECOM (NMP-CT-2006-016434) for financial support. One of the authors (I.D.W.S) is a Royal Society University Research Fellow and P.L.B. is the recipient of an Australian Research Council Federation Fellowship (project number FF0668728).

[1] Howard W.E. 2004 Sci. Am. 29076

[2] Sun Y. ,Borek C. , Hanson K. , Djurovich P. J. , Thomson M. E., Brooks J. , Brown J. J., Forrest S. R. 2007 Appl Phys Lett 90213503

[3] Lo S.-C. , Anthopoulos T. D. ,Namdas E. B., Burn P. L., Samuel I. D. W. 2005 Adv. Mater. 171945.

[4] Markham J. P. J. , Samuel I. D. W. , Lo S.-C. , Burn P. L. , Weiter M. , Bässler H. 2004 J. Appl. Phys. 95438.

[5] Anthopoulos T. D. , Markham J. P. J. , Namdas E. B., Samuel I. D. W. , Lo S.-C. , Burn P. L. 2003 Appl. Phys. Lett. 824824.

[6] Lo S.-C. , Male N. A. H. , Markham J .P. J. ,Magennis S. W. , Burn P. L., Salata O. V., Samuel I. D. W. 2002 Adv. Mater. 14975

[7] Markham J. P. J. , Lo S.-C., Magennis S. W. , Burn P. L. , Samuel. I. D. W. 2002 Appl. Phys. Lett. 802645.

[8] Braun S., Osikowicz W., Wang Y., Salaneck W. 2007 Org. El. 814

[9] Knights K. A. , Stevenson S. G. , Shipley C. P. , Lo S.-C. , Olsen S. , Harding R. E , Gambino S., Burn P. L., Samuel I. D. W. 2008 J. Mater. Chem. 182121

[10] Hwang J. ,Wan A., Kahn A. Mater. Sci. and Eng. 2009 R 641

[11] Koch N. , Kahn A. , Ghijsen J. , Pireaux J.-J.,Schwartz J. , Johnson R. L., Eischner A. 2003 Appl. Phys. Lett. 8270

[12] Grobosch M. ,Knupfer M. 2007 it Adv. Mater. 19754

[13] Walker A. B.,Kambili A. , Martin S. J. 2002 J. Phys. Cond. Matt. 149825

[14] Brütting W.,Verleb S. , Mückel A. 2001 Organic El. 21

[15] Lacic S. , Ingänas O. 2005 J. Appl. Phys. 97124901

[16] Scott J. C. , Malliaras G. G. , Chen W. D., Breach J.-C., Salem J. R., Brock P. J. ,Sachs S. B. , Chidseya C. E. D. 1999 Appl. Phys. Lett. 741510

[17] Davids P. S., Campbell I. H. , Smith D. L. 1997 J. Appl. Phys. 826319

[18] Scott J. C., Malliaras G. G. 1999 Chem. Phys. Lett 299115.

[19] Khan R. U. A. , Bradley D. D. C. , Webster M. A. ,Auld J. L. , Walker A. B. 2004 Appl. Phys. Lett. 84921

[20] Martin S. J., Walker A. B., Campbell A. J., Bradley D. D. C., J. Appl. Phys. 98 (2005) 063709.

[21] Gambino S., Stevenson S. G., Knights K. A., Burn P.L., Samuel I. D. W. 2008 Adv. Func. Mater. 19317.

[22] Wu C. C., Wu C. I. , Sturm J.C. , Kahn A. 1997 Appl. Phys. Lett. 701348

[23] Furukawa K. ,Terasaka Y. ,Ueda H. ,Matsumura M. 1997 Synth. Met. 9199

[24] Greczynski G. , Kugler T., Salaneck W. R. 2000 J. Appl. Phys., 12, 7187

[25] Crispin X. , Geskin V. ,Crispin A , Cornil J., Lazzaroni R. , Salaneck W. R., Bredas. J-L. 2002 J. Am. Chem. Soc. $\mathbf{1 2 4} 8131$

[26] Osikowicz W. , de Jong M. P. , Braun S. ,Tengstedt C. ,Fahlman M. , Salaneck W. R. 2006 Appl. Phys. Lett. 88193504

[27] J H T Williams, PhD Thesis, University of Bath, unpublished (2008)

[28] P. A. Tipler, R. A. Llewellyn, Modern Physics, 3rd edition, W.H. Freeman,(New York, 1999).

[29] Thakur A. K., Mukherjee A. K. ,Preethichandra D. M. G. , Takashima W. , Kaneto K. 2007 J. Appl. Phys. 101104508.

[30] Crone B. K. ,Davids P. S. ,Campbell I. H. , Smith D. L. 1998 J. Appl. Phys. 84833

[31] Murata K. ,Cinà S., Greenham N. C. 2001 Appl. Phys. Lett. 791193 